\title{
Large-Bowel Obstruction Secondary to Localized Rectal Giant Pseudopolyposis Complicating Ulcerative Colitis: First Reported Case
}

Localized giant pseudopolyposis is a rare complication of ulcerative colitis and Crohn's disease [1]. However, the formation of pseudopolyps in ulcerative colitis, is more common. Incidence rates reported vary from $12.5 \%$ to $74 \%[2,3]$.

A 68-year-old man with a 25-year history of pancolonic quiescent ulcerative colitis presented with acute large bowel obstruction. Preceding this, he had a 4month history of tenesmus, progressive "constipation" and mucoid discharge per rectum. Endoscopic views revealed almost complete luminal obstruction, with a large polypoid lesion high in the rectum (Figure 1). The colonoscopy could be completed only by using a paediatric colonoscope. Multiple pseudopolypoid lesions of the left and transverse hemicolon were observed (Figure 2 ), along with the macroscopic mucosal changes to the caecum associated with chronic ulcerative colitis. Figure 3 shows the high-resolution magnification chromoscopy appearance of the rectal lesion following chromoscopic enhancement with $2 \%$ indigo carmine solution (CF240ZI magnification endoscope; Olympus, Hamburg, Germany). Type II and IIIL crypt foci [4] or pit patterns are clearly demonstrable with a clear mucosal interface margin. The histological

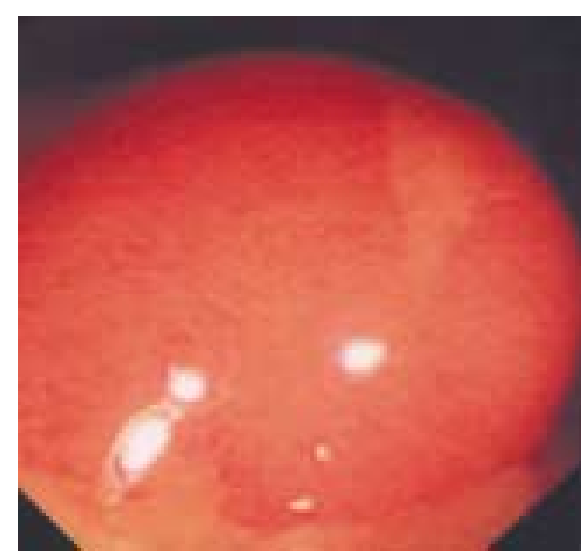

Figure 1 Obstructing giant pseudopolyp of the rectum complicating long-standing panulcerative colitis. findings (reported by two independent histopathologists), following combined endoscopic piecemeal and mucosal resection, were compatible with giant pseudopolyposis.

To our knowledge, this case represents the first report of a rectal giant pseudopolyp complicating ulcerative colitis. Additionally, the macroscopic appearances challenge the classical description of such lesions, which have previously been reported as predominantly frond-like, or villous aggregates $[1,5]$.

\section{Acknowledgement}

Dr Hurlstone is a Smith and Nephew Endoscopy Research Fellow.

\section{P. Hurlstone}

Gastroenterology and Liver Unit, Royal Hallamshire Hospital,

Sheffield, UK

\section{References}

${ }^{1}$ Foster DR. Localised giant pseudo-polyposis in ulcerative colitis. Postgrad Med J 1982; 58: $708-710$

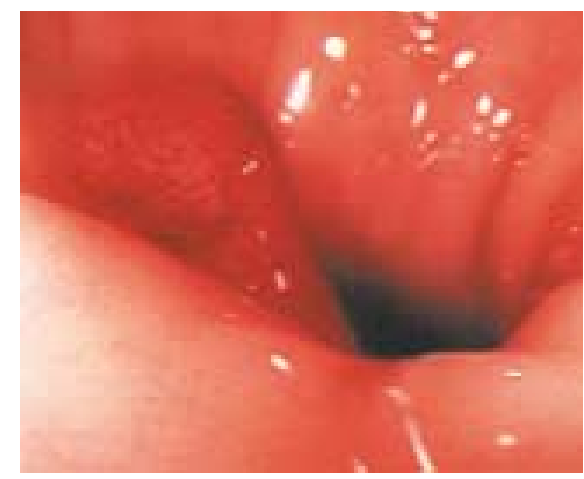

Figure 2 A further pseudopolypoid lesion in the transverse colon. Multiple pseudopolypoid lesions were present throughout the left and transverse hemicolon. Complete loss of the normal vascular architecture is noted additionally, in keeping with quiescent ulcerative colitis.
${ }^{2}$ De Dombal FT, Watts J, Watkinson G, Goligher JC. Local complications of ulcerative colitis: stricture, pseudo-polyposis and carcinoma of colon and rectum. Br Med J 1966; 5501: 1442 - 1447

${ }^{3}$ Jalan KN, Sircus W, Walker RJ, McManus JP. Pseudo-polyposis in ulcerative colitis. Lancet 1969; 2: 555-559

${ }^{4}$ Fuji T, Nagata K, Saito Y et al. The correspondence of magnifying endoscopic diagnosis and pathological diagnosis. Stomach Intest 1999; 34: 1653 - 1664

${ }^{5}$ Keating JW, Mindell HJ. Localised giant pseudo-polyposis in ulcerative colitis. Am J Roentgenol 1976; 126: 1178 - 1180

\section{Corresponding Author}

\section{P. Hurlstone, M.B. Ch.B.}

Room P14, Ward P2

Gastroenterology and Liver Unit

Royal Hallamshire Hospital

Sheffield

South Yorkshire

United Kingdom

Fax: $\quad+44-114-2712692$

E-mail: dphurlstone@doctors.org.uk

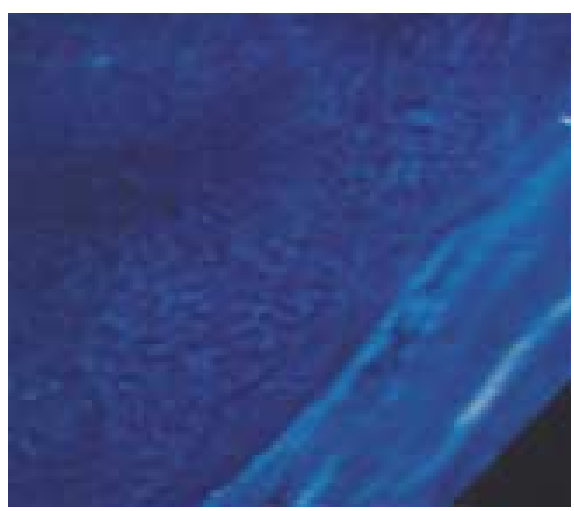

Figure 3 High-magnification $(\times 80)$ image of rectal giant pseudopolyp following chromoscopic enhancement with $2 \%$ indigo carmine wash. Type II and IIIL crypt foci are evident. 